\title{
Neuroimaging and Study Paradigms to Measure Infant Cognitive Ability
}

\author{
Seongkyung Bae \\ Biomedicine Academies at Englewood
}

\begin{abstract}
Although cognitive and psychological science has made great strides towards understanding the structures and functions of the human brain, understanding how brains are formed during the developmental stage of infancy remains a complex problem. Unlike adults, human infants cannot verbally communicate with researchers, make explicit responses, or control their bodies. Given these limitations, developmental scientists have employed smart study paradigms and research technologies to better understand the developmental trajectories of cognition and social understanding in infancy. This study provides an overview of these methods, including looking time measurements and modern neuroimaging techniques, and discusses what these approaches teach us about the core knowledge that allows infants to better learn and interact with the world.
\end{abstract}

Keywords: Infants; Cognitive development; Core knowledge; Developmental psychology 